\title{
Serum and synovial fluid concentrations of interleukin-18 and interleukin-20 in patients with osteoarthritis of the knee and their correlation with other markers of inflammation and turnover of joint cartilage
}

\author{
Michał Waszczykowski ${ }^{1}$, Anna Fabiś-Strobin², Igor Bednarski ${ }^{3}$, Joanna Narbutt ${ }^{3}$, Jarosław Fabiśs
}

\author{
1Department of Arthroscopy, Minimally Invasive Surgery, and Sports Traumatology, \\ Medical University of Lodz, Lodz, Poland \\ ${ }^{2}$ Clinic of Orthopaedic and Traumatology, Polish Mother's Memorial Hospital Research \\ Institute, Lodz, Poland \\ ${ }^{3}$ Dermatology, Paediatric Dermatology, and Oncology Clinic, Medical University \\ of Lodz, Lodz, Poland
}

Submitted: 30 March 2020; Accepted: 22 May 2020

Online publication: 3 July 2020

Arch Med Sci 2022; 18 (2): 448-458

DOI: https://doi.org/10.5114/aoms.2020.96717

Copyright @ 2020 Termedia \& Banach

\section{Abstract}

Introduction: Osteoarthritis $(\mathrm{OA})$ is the most common degenerative joint disease, and its aetiology is not entirely known. The aim of the study was to evaluate the involvement of interleukin-18 (IL-18) and interleukin-20 (IL-20) in the pathogenesis of knee OA and their correlations with other markers of inflammation and destruction of joint cartilage, as well as clinical and radiological changes.

Material and methods: The study included 25 patients with knee OA and a control group. The concentration of IL-18, IL-20, IL-6, MMP-1, MMP-3, COMP, PG-AG, and YKL-40 in serum and synovial fluid (SF) were determined. We also evaluated radiological lesions of the knee joint according to the Kellgren-Lawrence (K-L) scale, and clinical severity of the disease according to Western Ontario and McMaster Universities Osteoarthritis Index (WOMAC) and Lequesne Index.

Results: The concentrations of IL-18 and IL-20 were statistically significantly higher in serum of patients with OA than in the control group $(106.00$ $\pm 189.76 \mathrm{pg} / \mathrm{ml}$ vs. $16.73 \pm 16.99 \mathrm{pg} / \mathrm{ml}, p<0.001,17.69 \pm 13.45 \mathrm{pg} / \mathrm{ml}$ vs. $9.76 \pm 9.00 \mathrm{pg} / \mathrm{ml}, p<0.014)$. Serum concentration of IL-18 positively correlated with MMP-3 $(R=0.58 ; p=0.006)$ and YKL-40 $(R=0.48 ; p=0.002)$. The degree of radiological advancement of OA (K-L scale) correlated positively with clinical evaluation (WOMAC, $R=0.74, p \leq 0.001$; Lequesne Index, $R=0.57, p=0.003$.

Conclusions: The analysis of ROC curves showed that IL-20 as well as COMP, MMP-3, and YKL-40 may be diagnostic markers of knee OA. The observations indicate that IL-18 potentially mediates mainly in intra-articular processes and IL-20 could be primarily responsible for the systemic inflammatory reaction.

Key words: interleukin-18, interleukin-20, osteoarthritis, cartilage oligomeric matrix protein, biomarkers.

\section{Corresponding author:}

Michał Waszczykowski MD, PhD

Department of Arthroscopy,

Minimally Invasive

Surgery, and Sports

Traumatology

Medical University of Lodz

4 Kosciuszki St

90-419 Lodz, Poland

E-mail: mwaszczykowski@wp.pl 


\section{Introduction}

Osteoarthritis (OA) is the most common degenerative joint disease $[1,2]$. It is estimated that it affects about $10-20 \%$ of the population over 60 years of age [3-6]. It is characterised by a progressive process, which after years leads to the destruction of joints [7-10]. The leading symptoms are joint pain, swelling, limited range of motion, and progressive dysfunction leading to reduced physical fitness, social, family, sports, and professional activity. Despite the fact that numerous studies on the aetiopathogenesis of osteoarthritis have been conducted for many years, it has not been possible to determine its full course or all of its metabolic, biochemical, and immunoenzymatic pathways and processes [8-15]. Over many years, numerous substances and markers have been identified which participate in the cascade of inflammation and changes leading to the destruction of cartilage, but this still does not seem to be a complete list [5, 6, 16-22].

Interleukin 18 (IL-18) is part of the interleukin 1 (IL-1) superfamily and is produced by macrophages, chondrocytes, osteoblasts, fibroblasts, dendritic cells, neutrophils, and keratinocytes as an inactive form of 23-kDa protein. It is activated by one of the proteolytic enzymes - caspase-1 to a mature active form of $18-\mathrm{kDa}$ protein [2325]. IL-18 increases the chemotaxis of $T$ helper cells and stimulates their differentiation into $T$ helper 1 (Th1) and T helper 2 (Th2) cells. IL-18 also stimulates the immune system by enhancing the cytotoxicity of the natural killers (NK), stimulates interferon- $\gamma$ secretion, and enhances the chemotaxis of monocytes and neutrophils. IL-18 stimulates and activates macrophages, which through tumour necrosis factor $\alpha$ (TNF- $\alpha$ ) and interleukin $1 \beta$ (IL-1 $\beta$ ) secretion intensify the inflammatory process and destruction of joint cartilage. IL-18 also affects fibroblasts that increase the expression of intercellular adhesion molecule 1 (ICAM-1), chemokines, IL-8/CXCL8 and vascular cell adhesion molecule 1 (VCAM-1), and vascular endothelial growth factor (VEGF), which influences inflammatory response progression and angiogenesis [23-25]. In its structure IL-18 is similar to IL-1 $\beta$, which is a key cytokine responsible for the inflammatory process of the synovial membrane in patients with $\mathrm{OA}$, and it stimulates the activation of metalloproteinases [24]. The essence of IL-18 action in OA is its secretion by chondrocytes stimulated by IL-1 $\beta$. Like IL-1 $\beta$, it stimulates the inflammatory response in the synovial membrane and chondrocytes $[16,24,26]$. A few recent studies have indicated that it may inhibit the synthesis of proteoglycans, aggrecan, and type II collagen in chondrocytes $[7,16]$ and may additionally induce apoptosis and inhibit chondrocyte prolif- eration. In patients with rheumatoid arthritis (RA) IL-18 positively influences angiogenesis, which additionally stimulates the degradation of joint cartilage [25].

Interleukin 20 (IL-20) belongs to the interleukin 10 (IL-10) family, which includes: IL-10, IL-19, IL-20, IL-22, IL-24, and IL-26, and has a complex of two receptors: IL-20R1/IL-20R2 and IL-22R1/IL$20 R 2$ [27]. IL-20 is a cytokine with strong chemotactic and proinflammatory effects; it positively influences angiogenesis and apoptosis, affecting fibroblasts of the synovial membrane, endothelial cells, keratinocytes, and kidney epithelial cells. IL20 is secreted mainly by fibroblasts, macrophages, and granulocytes $[28,29]$. This interleukin increases the migration of neutrophils and T cells, thus increasing the inflammatory response [29]. In osteoarthritic synovial fibroblasts (OASFs), IL-20 has a stimulating effect on TNF- $\alpha, \mathrm{IL}-1 \beta$, and metalloproteinases 1 and 13 (MMP-1 and MMP-13) secretion [28]. IL-20 also affects chondrocytes by increasing the activity of interleukin 6 (IL-6) and MMPs, but it also inhibits the expression of transforming growth factor $\beta$ (TGF- $\beta$ ) and bone morphogenetic protein 2 (BMP-2), and the ability to synthesise aggrecan [28]. Recent studies have demonstrated its role in the aetiopathogenesis of psoriasis, psoriatic arthritis, rheumatoid arthritis (RA), atherosclerosis, myocardial infarction, stroke, prostate cancer, and renal failure [28-37].

Recent studies indicate that IL-20 plays an important role in RA pathogenesis, showing its influence on RA synovial fibroblasts (RASFs) for IL-6, interleukin 8 (IL-8) secretion, and chemotactic effects on neutrophils [30].

However, there are few data on the contribution of these interleukins to the aetiopathogenesis of OA and their role in the transformation and destruction of joint cartilage and the subchondral layer of bone. Recent studies by Hsu et al. indicate that IL-20 may participate in IL-1 $\beta$, TNF $\alpha$, MMP-1, MMP-3, and MMP-13 induction and inhibit the synthesis of aggrecan and type II collagen in cartilage, thus playing an important role in the pathogenesis of degenerative joint disease [28].

So far, however, there have been no studies or observations showing mutual correlations between IL-18 and IL-20 concentration in serum and synovial fluid (SF), expression of other inflammatory process markers and cartilage destruction, as well as clinical and radiological symptoms of OA.

The aim of this study was to demonstrate the role of IL-18 and IL-20 in the pathogenesis of idiopathic knee OA by evaluating their concentration in serum and SF, their correlation with other proinflammatory cytokines in serum and SF: IL-6, MMP-1, and MMP-3 and markers of joint cartilage 
destruction: cartilage oligomeric matrix protein (COMP), human cartilage glycoprotein (YKL-40), aggrecan (PG-AG), and the degree of radiological and clinical progression of the disease process expressed in Kellgren-Lawrence, Western Ontario and McMaster Universities Osteoarthritis Index (WOMAC), and Index of Severity for Osteoarthritis of the Knee (Lequesne index).

\section{Material and methods}

\section{Study group}

The study included 25 patients treated in the Department of Arthroscopy, Minimally Invasive Surgery, and Sport Traumatology of the Medical University of Lodz and the Orthopaedic Outpatient Clinic at the clinic, in whom the diagnosis of osteoarthritis (OA) of the knee joint was made based on the following symptoms: pain, swelling of the knee joint, crackling, and limitation of the range of joint movements, based on the criteria of the American College of Rheumatology [38]. Patients with history of immunological diseases (RA, PsA, Crohn's disease), neoplastic diseases, as well as recent or past significant knee joint injuries were excluded from further analysis.

The control group consisted of persons corresponding to age and gender distribution for the study group, in whom no clinical and radiological symptoms of degenerative disease were found and no other criteria were found to exclude them from the study.

All patients and control group members gave their informed and written consent to participate in the study. The study was conducted in accordance with the Declaration of Helsinki, and the protocol was approved by the local Bioethics Committee of the Medical University of Lodz, Poland (consent no. RNN/36/06/KB).

\section{Clinical evaluation}

The clinical evaluation of patients and the stage of disease progression was based on the Western Ontario and McMaster Universities Osteoarthritis Index (WOMAC) and LEQUESNE Index of Severity for Osteoarthritis of the Knee [39-42]. The WOMAC Scale is currently the most common tool for functional assessment of OAAO patients according to the guidelines of the American College of Rheumatology. The form consists of three parts: pain, stiffness, and function. The higher the score, the worse the function and the greater the pain in the occupied joint [40-42]. The Lequesne Scale consists of 11 questions in which the patient assesses and scores pain sensations, the distance they are able to walk, and the function of the limb expressed through everyday activities. The maximum score is 24 ; a score above 14 is ex- tremely severe. The Lequesne Index is currently recommended by the European League of Rheumatology (EULAR) for the assessment of osteoarthritis and its clinical severity, and the evaluation of treatment efficacy [39].

\section{Radiological assessment}

In each patient with OA, radiological images of the knee joint in a standing position in AP and lateral projection were taken. On the basis of radiological examinations, the degree of radiological advancement of the knee osteoarthritis was assessed using the criteria described by Kellgren and Lawrence [43]. Based on the described criteria for evaluation of radiological images, knee OA can be classified as grade 0 - no radiographic changes; grade I - doubtful space narrowing and possible marginal osteophytes; grade II - possible joint space narrowing and defined osteophytes observed; grade III - defined joint space narrowing, moderate multiple osteophytes, subchondral sclerosis and any possible deformity of bone contour; and grade IV - remarkable joint space narrowing, severe subchondral sclerosis, definite deformity of bone contour, and the presence of large osteophytes [43].

\section{Determination of the levels of IL-18 and IL-20 and other markers in serum}

Subsequently, IL-18, IL-20 and IL-6, MMP-1, MMP-3, COMP, YKL-40, and PG-AG serum concentration measurements were performed with enzyme-linked immunosorbent assay (ELISA) kits in the study and control group. Peripheral blood samples were collected from each patient in the morning. The samples were then centrifuged and serum samples were collected in $1.5 \mathrm{ml}$ Eppendorfs, which were then sealed, frozen, and stored at $-80^{\circ} \mathrm{C}$ for further immunoenzymatic testing. Subsequently, IL-18, IL-20 and IL-6, MMP-1, MMP-3, COMP, YKL-40, and PG-AG serum activity measurements were performed with enzyme-linked immunosorbent assay (ELISA) kits from R\&D Systems Europe, Ltd, Abingdon, UK (IL-6, IL-18, IL-20, MMP-1, MMP-3), BioVendor $\mathrm{GmbH}$, Heidelberg, Germany (COMP), Metra Quidel, San Diego, USA (YKL-40), and BioSource Europe S.A., Nivelles, Belgium (PG-EASIA), according to the manufacturers' instructions. The minimum detection level was determined as $0.7 \mathrm{pg} / \mathrm{ml}$ for IL-6, $4.57 \mathrm{pg} /$ $\mathrm{ml}$ for IL-18, $16.6 \mathrm{pg} / \mathrm{ml}$ for IL-20, $0.095 \mathrm{ng} / \mathrm{ml}$ for MMP-1, $0.045 \mathrm{ng} / \mathrm{ml}$ for MMP-3, $0.4 \mathrm{ng} / \mathrm{ml}$ for COMP, $10 \mathrm{ng} / \mathrm{ml}$ for YKL-40, and $0.9 \mathrm{ng} / \mathrm{ml}$ for PG-EASIA. The same activities (blood collection, centrifugation, freezing, and storage) were performed in the control group, followed by the same immunoenzymatic assays as in the study group using appro- 
priate ELISA test kits (R\&D Systems, BioVendor, Metra Quidel, BioSource).

The PG-AG concentration in the control group was not determined for technical reasons.

Determination of the level of IL-18, IL-20, and other markers in synovial fluid (SF)

The concentration of IL-18, IL-20 and IL-6, MMP-1, MMP-3, COMP, YKL-40, and PG-AG in SF was then measured using enzyme-linked immunosorbent assay (ELISA) tests. Synovial fluid (SF) was collected from patients in the study group (OA) during a therapeutic knee arthrocentesis. The joint puncture was performed under strictly sterile conditions, with 18-gauge needle to puncture the joint from the suprapatellar lateral access. The SF samples were then filtered, centrifuged at $4^{\circ} \mathrm{C}$ at $300 \mathrm{~g}$ for $15 \mathrm{~min}$, transferred to $1.5 \mathrm{ml}$ Eppendorfs, and then sealed, frozen, and stored at $-80^{\circ} \mathrm{C}$ for further immunoenzymatic determination. The activity of IL-18, IL-20 and IL-6, MMP-1, MMP-3, COMP, YKL-40, and PG-AG in SF was then measured using enzyme-linked immunosorbent assay (ELISA) tests with kits from R\&D Systems Europe, Ltd, Abingdon, UK (IL-6, IL-18, IL-20, MMP-1, MMP-3), BioVendor GmbH, Heidelberg, Germany (COMP), Metra Quidel, San Diego, USA (YKL-40), and BioSource Europe S.A., Nivelles, Belgium (PG-EASIA), according to the manufacturers' instructions. The minimum detection level was determined as $0.7 \mathrm{pg} / \mathrm{ml}$ for IL-6, $4.57 \mathrm{pg} /$ $\mathrm{ml}$ for IL-18, $16.6 \mathrm{pg} / \mathrm{ml}$ for IL-20, $0.095 \mathrm{ng} / \mathrm{ml}$ for MMP-1, $0.045 \mathrm{ng} / \mathrm{ml}$ for MMP-3, $0.4 \mathrm{ng} / \mathrm{ml}$ for COMP, $10 \mathrm{ng} / \mathrm{ml}$ for YKL-40, and $0.9 \mathrm{ng} / \mathrm{ml}$ for PG-EASIA.

Due to technical problems during the tests, the YKL-40 concentration in SF was determined only in 4 patients. For this reason, these results were not taken into account in the statistical analysis.

\section{Statistical analysis}

Baseline characteristics of participants including age, clinical scales, and laboratory findings were presented as means with standard deviations or medians with interquartile range. Distribution of continuous variables was evaluated using Shapiro-Wilk test. Normally distributed data were analysed with Welch's test and non-normally distributed data were analysed with Mann-Whitney $U$ test. Due to the presence of several outliers, correlations between each variable were examined using Spearman's rank correlation. A correlation coefficient ranging from 0.00 to 0.19 was considered as very weak, 0.20 to 0.39 as weak, 0.40 to 0.59 as moderate, 0.60 to 0.79 as strong, and 0.80 to 1.0 as very strong.

A $p$ value below 0.05 was deemed significant. Diagnostic performance of each biomarker in OA detection was assessed by ROC curve analysis and calculation Youden's Index and area under the curve. All analyses were made using Statistica 13 software.

\section{Results}

Twenty-five (25) patients $(60 \%$ of them were women) aged between 44 and 76 years (mean 59.88 years) were qualified for the analysis. The control group consisted of 32 healthy volunteers. There were no statistically significant differences between the two groups in terms of age and gender (Table I). We found a statistically significant increase in serum levels of both IL-18 and IL-20 in the examined patients with OA of the knee joint compared to the control group ( $p<0.001$ for IL-18 and $p<0.014$ for IL-20, respectively; Table I). Our study also showed that IL-6 serum levels in OA patients were statistically significantly higher than in control serum ( $p<0.001$; Table I). However, among the examined metalloproteinases (MMP-1, MMP-3), only MMP-3 showed statistically significantly higher serum level in OA patients compared to the control group ( $p<0.002$; Table I).

In our study we also analysed the concentrations of markers of turnover and destruction of joint cartilage in serum of patients with OA knee joint and compared them with their level in the control group. We showed that both YKL-40 and COMP showed significantly higher serum concentrations in the study group compared to control $(p<0.001$; Table I).

We also found that the serum level of IL-18 in the study group positively correlated with the level of MMP-3 $(R=0.52)$ and YKL-40 $(R=0.48)$ (Table II). The serum concentration of MMP-3 in the examined patients correlated positively with YKL-40 and IL-6 (Table II). Our analysis also indicated that serum IL-6 correlates positively with YKL-40 (Table II). We also found significant positive correlations between serum concentration of markers of joint cartilage destruction: COMP with YKL-40 and COMP with PG-AG (Table II). The concentration of IL-20 in the synovial fluid of patients with $\mathrm{OA}$ of the knee joint positively correlated with the concentration of MMP-1 (Table III).

There were no significant correlations between the radiological stage of disease progression expressed in Kellgren-Lawrence scale and IL-18 and IL-20 and any other studied markers of inflammation and cartilage destruction in serum. Such a correlation was found in the SF of the examined patients, where PG-AG concentration positively correlated with radiological intensity of degenerative changes on the K-L scale (Table III). Our analysis also indicated that the degree of degenerative changes on the K-L scale positively correlated with the degree of clinical severity of the disease on 
Table I. Clinical parameters and serum concentrations of metalloproteinases, inflammatory cytokines, and cartilage turnover markers in the control and study group

\begin{tabular}{|c|c|c|c|}
\hline Parameter & Osteoarthritis $(N=25)$ & Control group $(N=32)$ & $P$-value \\
\hline Mean age [years] ${ }^{*}$ & $59.88 \pm 12.08$ & $55.75 \pm 8.69$ & 0.157 \\
\hline Kellgren-Lawrence Grading $(0-4)^{\star *}$ & $3(2,4)$ & - & - \\
\hline WOMAC score $(0-100)^{*}$ & $46.42 \pm 8.93$ & - & - \\
\hline Lequesne index $(0-24)^{*}$ & $10.31 \pm 2.29$ & - & - \\
\hline MMP-1 $[\mathrm{ng} / \mathrm{ml}]^{*}$ & $4.81 \pm 2.49$ & $7.76 \pm 3.74$ & 0.001 \\
\hline MMP-3 $[\mathrm{ng} / \mathrm{ml}]^{*}$ & $23.34 \pm 29.22$ & $9.18 \pm 3.06$ & 0.002 \\
\hline PG-AG $[\mathrm{ng} / \mathrm{ml}]^{*}$ & $4689.32 \pm 518.47$ & - & - \\
\hline IL-6 [pg/ml] ${ }^{*}$ & $7.11 \pm 21.02$ & $5.50 \pm 2.22$ & $<0.001$ \\
\hline IL-18 [pg/ml] $]^{*}$ & $106.00 \pm 189.76$ & $16.73 \pm 16.99$ & $<0.001$ \\
\hline YKL-40 [ng/ml] ${ }^{*}$ & $88.98 \pm 72.29$ & $44.31 \pm 17.50$ & $<0.001$ \\
\hline IL-20 [pg/ml] $]^{\star}$ & $17.69 \pm 13.45$ & $9.76 \pm 9.00$ & 0.014 \\
\hline COMP [ng/ml] & $2299.56 \pm 730.10$ & $835.85 \pm 434.62$ & $<0.001$ \\
\hline
\end{tabular}

both the WOMAC and LEQUESNE INDEX scales (Table II). However, there were no strong correlations between the clinical and functional stage of knee joint degeneration (WOMAC, LEQUESNE INDEX) and serum markers. However, we indicated such correlations in the SF of the study group, where IL-20 activity positively correlated with LEQUESNE results, and MMP-1 and MMP-3 activity positively correlated with WOMAC score (Table III).

Table II. Significant Spearman's correlation coefficients between studied clinical parameters and inflammatory/cartilage biomarkers in serum

\begin{tabular}{|lcc|}
\hline Pair of parameters $(N=25)$ & $R$ & $P$-value \\
\hline WOMAC vs. LI & 0.92 & $\leq 0.001$ \\
\hline Age vs. KL & 0.86 & $\leq 0.001$ \\
\hline KL vs. WOMAC & 0.74 & $\leq 0.001$ \\
\hline Age vs. WOMAC & 0.66 & $\leq 0.001$ \\
\hline MMP-3 vs. YKL-40 & 0.65 & 0.002 \\
\hline MMP-3 vs. IL-6 & 0.58 & 0.006 \\
\hline KL vs. LI & 0.57 & 0.003 \\
\hline Age vs. LI & 0.57 & 0.003 \\
\hline YKL-40 vs. COMP & 0.54 & 0.017 \\
\hline IL-6 vs. YKL-40 & 0.53 & 0.016 \\
\hline MMP-3 vs. IL-18 & 0.52 & 0.016 \\
\hline PG-AG vs. COMP & 0.50 & 0.025 \\
\hline IL-18 vs. YKL-40 & 0.48 & 0.031 \\
\hline LI vs. MMP-1 & 0.43 & 0.050 \\
\hline
\end{tabular}

In our study, we also analysed the concentration of individual biomarkers in serum and SF of the examined patients (Table IV). We found that in all examined markers their levels in SF fluid are statistically significantly higher than in serum (Table IV). Analysing these relationships we found that among some markers the ratio of their levels in SF to serum is many times higher than between others (Fold change, Table IV).

ROC analysis was performed to establish the diagnostic performance of each biomarker. COMP showed the highest sensitivity and specificity (AUC $=0.967$, Youden's Index 0.87, cut-off value $1827.81 \mathrm{ng} / \mathrm{ml}$ ). Also, we found that IL-20, MMP-3, and YKL-40 have significant sensitivity and specificity in OA detection (IL-20: AUC = 0.715, Youden's Index 0.48, cut-off value $7.5 \mathrm{ng} / \mathrm{ml}$; MMP-3: AUC $=0.758$, Youden's Index 0.43, cut-off value $10.9 \mathrm{ng} / \mathrm{ml}$; YKL-40: AUC = 0.779, Youden's Index 0.53 , cut-off value $47.40 \mathrm{ng} / \mathrm{ml}$ ) (Figures 1, 2).

Table III. Significant Spearman's correlation coefficients between studied clinical parameters and inflammatory/cartilage biomarkers in synovial fluid

\begin{tabular}{|lcc|}
\hline Pair of parameters & $\boldsymbol{R}$ & $P$-value \\
\hline MMP-1 vs. IL-20 & 0.72 & 0.004 \\
\hline WOMAC vs. MMP-3 & 0.70 & 0.016 \\
\hline WOMAC vs. MMP-1 & 0.53 & 0.049 \\
\hline LI vs. IL-20 & 0.52 & 0.045 \\
\hline KL vs. PG-AG & -0.56 & 0.030 \\
\hline Age vs. IL-6 & -0.56 & 0.030 \\
\hline
\end{tabular}


Table IV. Inflammatory and cartilage biomarkers in serum and synovial fluid in OA patients

\begin{tabular}{|lcccc|}
\hline Variable & Serum $^{*}$ & Synovial fluid & Fold change & $P$-value \\
\hline MMP-1 [ng/ml] & 4.83 & 45.32 & 9.39 & $\leq 0.001$ \\
\hline MMP-3 [ng/ml] & 23.06 & 414.12 & 17.96 & $\leq 0.001$ \\
\hline PG-AG [ng/ml] & 4689.32 & 87653.73 & 18.69 & $\leq 0.001$ \\
\hline IL-6 [pg/ml] & 7.01 & 843.17 & 120.28 & $\leq 0.001$ \\
\hline IL-18 [pg/ml] & 106.00 & 549.49 & 5.18 & 0.0001 \\
\hline IL-20 [pg/ml] & 17.80 & 34.94 & 1.96 & 0.0281 \\
\hline COMP [ng/ml] & 2315.61 & 15822.69 & 6.83 & $<0.001$ \\
\hline
\end{tabular}

*The values are given as a mean.

A

IL-20 AUC $=0.715$, Youden's Index $=0.48$ Cut-off value $=7.50 \mathrm{ng} / \mathrm{ml}$

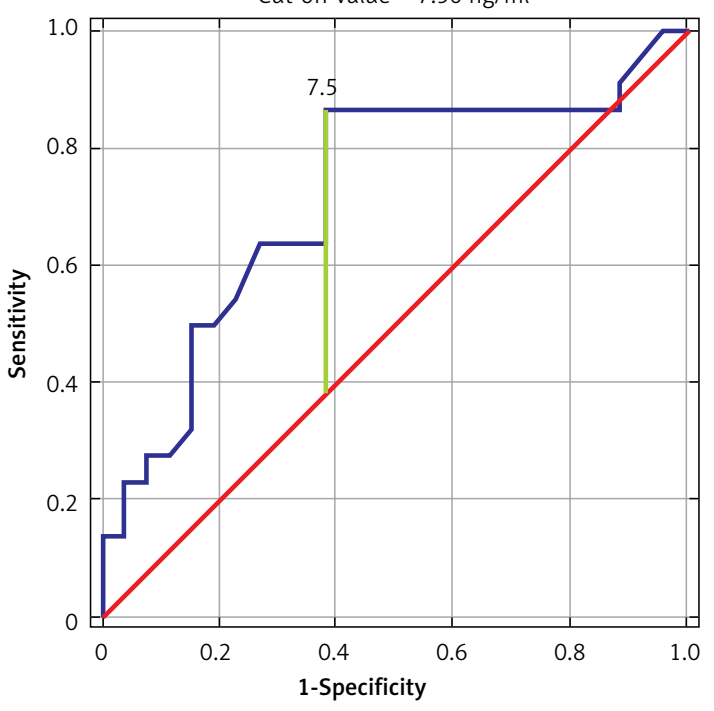

B

COMP

AUC $=0.967$, Youden's Index $=0.87$

Cut-off value $=1827.81$

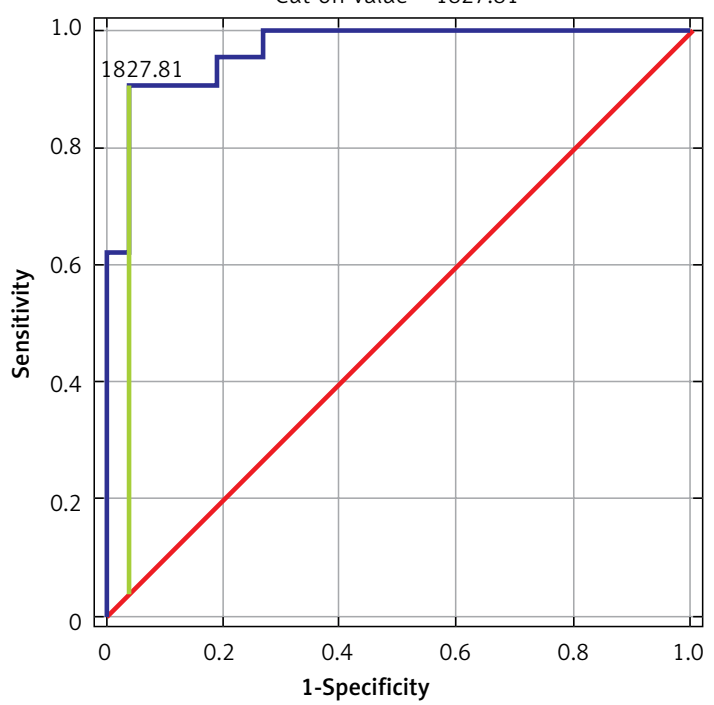

Figure 1. ROC curve analysis for IL-20 (A) and for COMP (B)

A

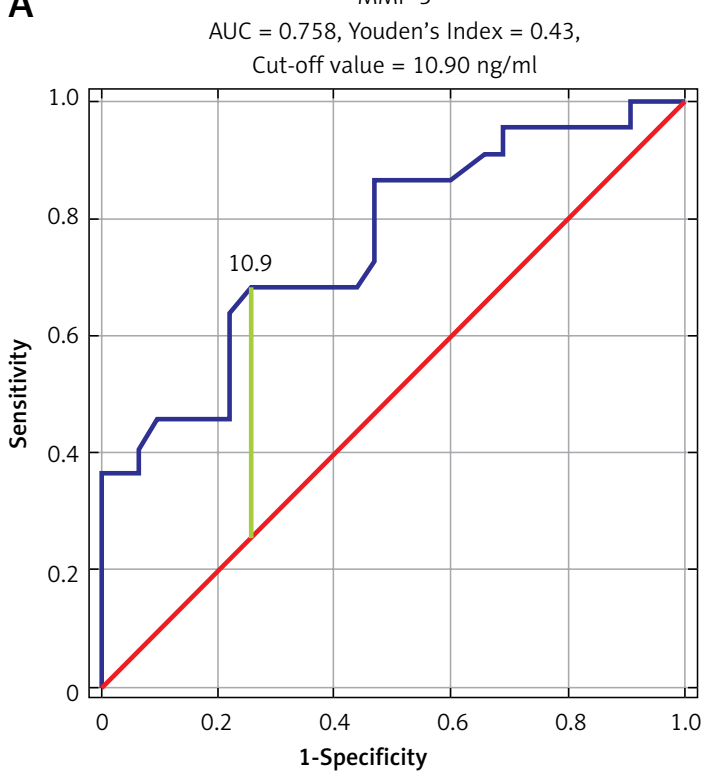

B

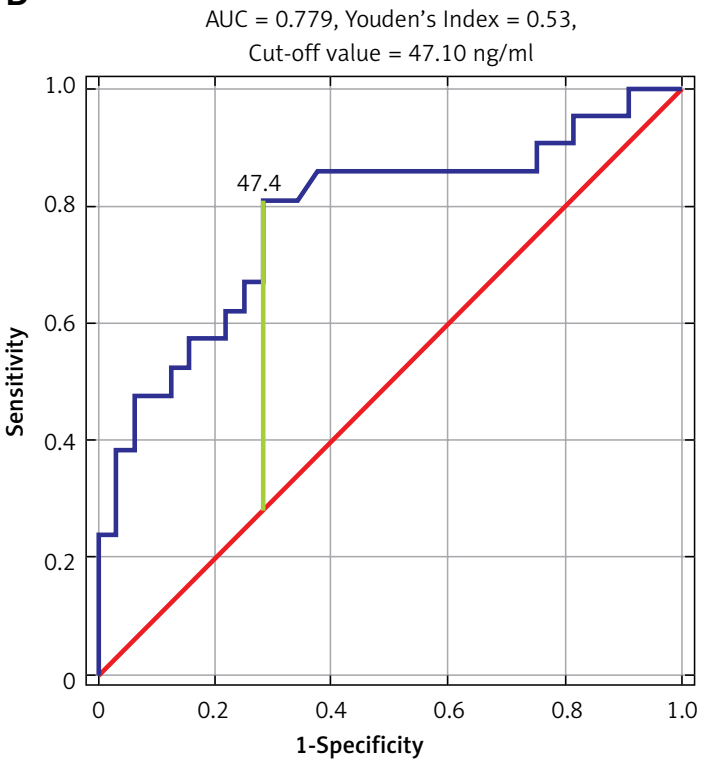

Figure 2. ROC curve analysis for MMP-3 (A) and for YKL-40 (B) 


\section{Discussion}

Our knowledge and analysis of literature indicate that this study might be the first one to present in such a broad scope the contribution of IL-18 and IL-20 to the pathogenesis of inflammation and destruction of joint cartilage in OA of the knee and its correlation with radiological parameters of progression of lesions (Kellgren-Lawrence scale), other markers of inflammation and destruction of joint cartilage, and functional assessment of clinical condition (WOMAC index, LEQUESNE index). Measurements and analyses of both serum and SF indicate that both IL-18 and IL-20 may play an important role in the process of knee degenerative changes, and their concentrations were statistically significantly higher than in the control group (Table I). Moreover, we found the following study demonstrated positive correlations between IL-18 level in serum of patients with knee joint osteoarthritis and MMP-3 concentration. This observation may indicate that IL-18 positively influences the expression of this metalloproteinase by intensifying the collagen chain destruction processes, thus stimulating the processes of joint cartilage destruction. Similar conclusions were formulated in the study by Dai et al. They revealed a positive correlation between the increase in expression of metalloproteinases and aggrecanase 2 in chondrocytes with IL-18 [24]. In addition, Fu et al. pointed out the strongly inductive character of increased IL-18 expression on OA knees by intensifying the synthesis of metalloproteinases in chondrocytes [26]. The significant correlation between MMP-3 serum level and IL-6 level (Table II) proves that together with IL-18 they stimulate catabolic processes of collagen chains of joint cartilage. Moreover, studies by Ruan et al. show that in addition to the documented relationship between IL-18 and MMP-3, the serum level of IL-18 correlates positively with the concentration of MMP-13, which additionally indicates a multimolecular positive effect of this interleukin on the processes of collagen and proteoglycan destruction in joint cartilage [16, $24,26]$. The above conclusion is confirmed by the positive correlation of IL-18 serum concentration

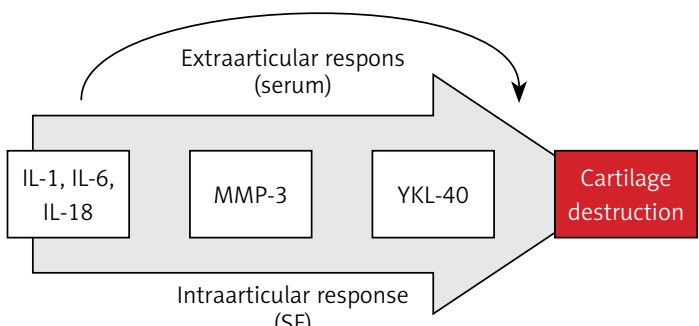

Figure 3. Interleukin-18-dependent pathway of osteoarthritic cartilage turnover

IL-1-interleukin 1, IL-6-interleukin 6, IL-18-interleukin 18, MMP-3-metalloproteinase 3, YKL-40 - human cartilage glycoprotein 39, SF - synovial fluid. with YKL-40, documented in our material, and additionally an analogous correlation in the case of IL-6 (Table II), revealed earlier by studies of Volck et al. [22]. This may indicate a complex and multi-track mechanism leading to the final destruction of the joint cartilage and the progression of degenerative changes. This may be confirmed by Smeets et al. who, in a collagen-induced arthritis animal model of $\mathrm{OA}$ in mice, demonstrated that the blockage of IL-18 action by administration of binding protein equivalent results in milder and less symptomatic course and picture of arthritis and destruction of joint cartilage [44]. It seems that IL-18 neutralisation inhibits the important catabolic pathway of joint cartilage by blocking the influence of serum IL-18 activity on the synthesis of aggrecan in knee cartilage chondrocytes, documented by Inoue et al. in experimental studies on mice [45]. The results of the above studies and our own research indicate the intra- and extra-articular mechanism of cartilage destruction activated by IL-18. Both mechanisms are associated with the activation of the synthesis of metalloproteinases and aggrecanases by synoviocytes and fibroblasts of synovial membrane and chondrocytes, while the extra-articular mechanism is an increase in the presence of IL-18 serum. The above observations suggest the presence of a previously undescribed mechanism of the vicious circle of degenerative lesion development consisting of increased IL-18 secretion in the joint causing its secondary growth in the blood serum with which it again reaches the synovial membrane, potentially providing an additional stimulation to the catabolic processes of the articular cartilage (Figure 3).

In our study we also found statistically significantly higher IL-20 concentration in OA serum than in the control group (Table I). Our results are consistent with those published in recent years. Hsu et al. pointed out the important role that IL-20 plays in the pathogenesis of OA in a rodent OA model [28]. They demonstrated the expression of both IL-20 and its receptors in the fibroblasts of the synovial membrane (OASFs) and chondrocytes (OACCs) of patients with OA. Moreover, they proved that IL-20 positively influences the synthesis of metalloproteinases (MMP-1, MMP-13), $\mathrm{IL}-1 \beta$, and IL- 6 in OASFs and OACCs. By acting on OACCS, IL-20 inhibits collagen synthesis, reducing the activity and level of aggrecan (PG-AG). It is also confirmed by our results, which indicate a positive correlation between the concentration of IL-20 in SF and the level of MMP-1 (Table III).

Scrivo et al. in their analysis compared the activity of IL-20 in groups of patients with OA, RA, and PsA. They showed that the increase in IL-20 activity is associated with its pro-inflammatory effect but is mainly manifested in a systemic response [46]. 
In our work we also tried to demonstrate whether the concentration of IL-18 and IL-20 and other biomarkers of inflammation and cartilage destruction correlates in a statistically significant way with the radiological severity of degenerative changes. In order to evaluate the radiological changes in the knee joints of patients with OA, we used the Kellgren-Lawrence scale, which is a commonly used and recommended scale for radiological assessment of OA in the knee joint. We found a strong negative correlation between the level of aggrecan (PG-AG) in the SF and the radiological degree of degenerative changes (K-L scale; Table III). Monibi et al. analysed the activity of various inflammatory mediators (IL-6, IL-8, MMP-1, MMP-2) in $\mathrm{SF}$ in a group of patients with knee joint $\mathrm{OA}$ [47]. They, and the other authors, found that the level of IL- 6 and IL- 8 in the SF correlates with the degree of radiological progression of $\mathrm{OA}$ in the knee joint expressed in the K-L scale [41, 47-49]. Similarly, Bellucci et al. found a positive correlation between the Kellgren-Lawrence score and the activity of bradykinin (BK) and IL-6 in the examined SF [50]. They also confirmed our observations on the participation and mutual influence of cytokines and metalloproteinases on the intensity of inflammatory process in OA and on the processes of joint cartilage destruction [50]. They showed a positive correlation between the activity of MMP-1 and MMP-3 and the level of COMP in SF. Although the concentration of COMP in SF was statistically significant, it did not correlate with the degree of radiological severity of degenerative changes on the Kellgren-Lawrence scale.

In our analysis, we also demonstrated that the degree of radiological severity of degenerative lesions (K-L scale) may positively correlate with the degree of clinical severity of the disease process expressed by both WOMAC and LEQUESNE INDEX scales (Table II). We also found that IL-20 concentration in SF positively correlates with the LEQUESNE INDEX. Also, MMP-1 and MMP-3 levels in SF positively correlate with clinical severity of OA expressed in WOMAC (Table III). It seems that our observations are confirmed by the study of Bellucci and Meini, who showed strong correlation between COMP level in SF and clinical evaluation of the severity of lesions in OA expressed in the LEQUESNE INDEX scale [50].

In our study we found that in all studied markers the concentration was statistically significantly higher in SF than in serum (Table IV). This may indicate a strong local (intra-articular) effect of these biomarkers' pathways on the degradation of the joint cartilage. Compared the ratio of the activity of markers of joint cartilage destruction (COMP, PG-AG) in SF to serum, we found that their concentrations in SF were many times higher (6.83 for
COMP and 18.69 for PG-AG, respectively; Table IV). Similar observations were made by El-Arman et al. [51]. They also showed statistically significant elevation of COMP and aggrecan levels in serum and SF in patients with OA of the knee [51]. The level of COMP and aggrecan activity in SF was statistically significantly higher than in serum, which indicates that the release of these markers is closely related to the cartilage degradation process in the joint [51-54]. However, it is interesting to observe the IL-18 and IL-20 concentration ratio (fold change) in SF to serum in patients with knee OA (Table IV). It indicates that IL-18 (fold change: 5.18) may play a greater role in the pathogenesis of osteoarthritis (OA) locally, within the joint, but IL-20 (fold change: $1.96)$ in systemic response. Moreover, significantly higher concentration of IL-6 in SF than in serum (fold change: 120) indicates its strong intra-articular activity (Table IV). The clear correlation of IL- 6 concentration with the intensity of radiological degree of degenerative changes (K-L scale) indicates its strong involvement in the processes of joint cartilage destruction occurring within the knee joint $[47,50]$.

Lastly, we decided to study whether each of studied biomarkers could serve as sensitive diagnostic tool in OA screening (Figures 1 and 2). It has been shown that COMP, as well as IL-20, YKL40, and MMP-3, could be the best candidates; however, more studies are needed to establish their role as an OA screening tool. We have shown that COMP could indicate that $O A$ is present. We found that the cut-off value for COMP was $1827.81 \mathrm{ng} / \mathrm{ml}$, which means that patients without any radiographic study could be suffering from OA. This biomarker needs to be evaluated in the future in terms of overlapping syndromes and suspicion of $\mathrm{OA}$ in patients without radiographic imaging confirmation. Lastly, it needs to be evaluated whether COMP is influenced by other factors. In our study we discovered positive moderate correlation between COMP and YKL-40, which leads to the possibility that serum levels of COMP are affected by YKL-40. As a biomarker YKL-40 performance was weaker compared to COMP, but given small sample size we are unable to establish meaningful conclusions and real significance of this finding. A similar observation and analysis were used in the study by Zivanovic et al. [55]. They used the ROC curve calculation method to verify the hypothesis that YKL-40 in serum of patients with OA may be a sensitive marker of degenerative disease symptoms in the form of osteophytic joint lesions observed in ultrasound [55].

Our work, therefore, confirms that IL-18 and IL-20 might play an important role in the pathogenesis of osteoarthritis. It also shows their influence on pro-inflammatory processes and 
degradation of collagen and joint cartilage. Their correlation with the concentration of other pro-inflammatory cytokines and metalloproteinases indicates that they may be important factors in the aetiopathogenesis of OA. Our observations, research results, and literature analysis show that IL-18 may be a key factor in the cascade of biochemical and immunoenzymatic transformations occurring in the joint in which they are involved: IL-1, IL-6, IL-18, MMP-3, and YKL-40. This pathway eventually leads to collagen chain destruction and cartilage degradation (Figure 3 ). This may give the opportunity to search for new therapeutic possibilities of inhibition of biochemical processes induced and controlled by IL-18 and IL-20. This is confirmed by the reports of Hsu et al., Senolt et al., and Smeets et al. The first of them demonstrated that monoclonal antibodies directed against IL20 (7E) inhibit the expression of IL-1, IL-20, and metalloproteinases in the fibroblasts of the synovial membrane and inhibit the process of cartilage degradation and osteophytes formation [28, 32, 33]. Senolt et al. demonstrated a positive effect of monoclonal antibodies against IL-20 (NNC01090012) in the treatment of seropositive patients with RA [54]. Smeets et al. in the collagen-induced model of osteoarthritis in mice demonstrated that the inhibition of IL-18 action by administration of binding proteins causes milder and less symptomatic course and picture of arthritis and destruction of joint cartilage [44].

However, our work has a few weak points and limitations. The first one is the relatively small group of patients. This study, as well as the results, are preliminary and indicate the necessity and advisability of further research and analysis of the above parameters and biomarkers. The authors of this publication continue to conduct research and broaden the group of patients qualified for analysis. Another limitation is the lack of randomisation of the study group. Another weak point of our study is the lack of a control group for synovial fluid. The control group in our study consisted of healthy volunteers, so we could not collect SF. It also seems justified to perform in the future a wider range of immunoenzymatic tests to determine also the activity of anti-inflammatory markers (e.g. IL-4, IL-10, IL-13) and other markers of cartilage and bone destruction.

In conclusion, IL-18 and IL-20 can play an important role in the aetiopathogenesis of knee osteoarthritis. The observations indicate that IL-18 potentially mediates mainly in intra-articular processes and is responsible for the destruction of joint cartilage, which is indicated by positive correlations with the activity of MMP-3 and YKL-40. On the other hand, IL-20 could be primarily responsible for the systemic inflammatory reac- tion. Its activity positively correlated with clinical evaluation of patients on the LEQUESNE scale. It also seems that IL-20, as well as COMP, YKL-40 and MMP-3, may be a sensitive marker in the diagnosis of osteoarthritis. Our research might indicate the necessity of distinguishing additional extra-articular molecular pathway of intra-articular stimulation of knee cartilage destruction by IL- 6 and IL-18 as a result of their significant growth in the blood serum with which they reach the joint, thus activating the mechanism of the vicious circle. However, more research is needed in this field.

\section{Acknowledgments}

The study was supported by internal funds of the Medical University of Lodz, Lodz, Poland (grant number: 502-03/7-048-02/502-54-006; 503/1-040-02/ 503-51-001-1900).

\section{Conflict of interest}

The authors declare no conflict of interest.

\section{References}

1. Martel-Pelletier J, Barr AJ, Cicuttini FM, et al. Osteoarthritis. Nat Rev Dis Primers 2016; 2: 16072.

2. Johnson VL, Hunter DJ. The epidemiology of osteoarthritis. Best Pract Res Clin Rheumatol 2014; 28: 5-15.

3. Woolf AD, Pfleger B. Burden of major musculoskeletal conditions. Bull World Health Organ 2003; 81: 646-56.

4. Okuyan HM, Terzi MY, Ozcan O, Kalaci A. Association of UCMA levels in serum and synovial fluid with severity of knee osteoarthritis. Int J Rheum Dis 2019; 22: 1884-90.

5. Goldring MB. The role of cytokines as inflammatory mediators in osteoarthritis: lessons from animal models. Connect Tissue Res 1999; 40: 1-11.

6. Mieloch AA, Richter M, Trzeciak T, Giersig M, Rybka JD. Osteoarthritis severely decreases the elasticity and hardness of knee joint cartilage: a nanoindentation study. J Clin Med 2019; 8: 1865.

7. Wojdasiewicz P, Poniatowski ŁA, Szukiewicz D. The role of inflammatory and anti-inflammatory cytokines in the pathogenesis of osteoarthritis. Mediators Inflamm 2014; 2014: 561459.

8. Porée B, Kypriotou M, Chadjichristos C, et al. Interleukin-6 (IL-6) and/or soluble IL-6 receptor down-regulation of human type II collagen gene expression in articular chondrocytes requires a decrease of Sp1.Sp3 ratio and of the binding activity of both factors to the COL2A1 promoter. J Biol Chem 2008; 283: 4850-65.

9. Qiao YQ, Jiang PF, Gao YZ. Lutein prevents osteoarthritis through Nrf2 activation and downregulation of inflammation. Arch Med Sci 2018; 14: 617-24.

10. Sui Y, Lee JH, DiMicco MA, et al. Mechanical injury potentiates proteoglycan catabolism induced by interleukin- 6 with soluble interleukin-6 receptor and tumor necrosis factor alpha in immature bovine and adult human articular cartilage. Arthritis Rheum 2009; 60: 2985-96.

11. Kwan Tat S, Padrines M, Théoleyre S, Heymann D, Fortun Y. IL-6, RANKL, TNF-alpha/IL-1: interrelations in bone resorption pathophysiology. Cytokine Growth Factor Rev 2004; 15: 49-60. 
12. Chenoufi HL, Diamant M, Rieneck K, Lund B, Stein GS, Lian JB. Increased mRNA expression and protein secretion of interleukin- 6 in primary human osteoblasts differentiated in vitro from rheumatoid and osteoarthritic bone. J Cell Biochem 2001; 81: 666-78.

13. Monibi F, Roller BL, Stoker A, Garner B, Bal S, Cook JL Identification of synovial fluid biomarkers for knee osteoarthritis and correlation with radiographic assessment. J Knee Surg 2016; 29: 242-7.

14. Kim KS, Lee YA, Choi HM, Yoo MC, Yang HI. Implication of MMP-9 and urokinase plasminogen activator (UPA) in the activation of pro-matrix metalloproteinase (MMP)13. Rheumatol Int 2012; 32: 3069-75.

15. Nees TA, Rosshirt N, Zhang JA, et al. Synovial cytokines significantly correlate with osteoarthritis-related knee pain and disability: inflammatory mediators of potential clinical relevance. J Clin Med 2019; 8: 1343.

16. Ruan G, Xu J, Wang K, at al. Associations between knee structural measures, circulating inflammatory factors and MMP13 in patients with knee osteoarthritis. Osteoarthritis Cartilage 2018; 26: 1063-9.

17. Waszczykowska A, Goś R, Waszczykowska E, Dziankowska-Bartkowiak B, Jurowski P. Assessment of skin microcirculation by laser Doppler flowmetry in systemic sclerosis patients. Adv Dermatol Allergol 2014; 31: 6-11.

18. De Ceuninck F, Sabatini M, Pastoureau P. Recent progress towards biomarker identification in osteoarthritis. Drug Discov Today 2011; 16: 443-9.

19. El-Ahwany EG, Mourad L, Zoheiry MM, et al. MicroRNA-122a as a non-invasive biomarker for HCV genotype 4-related hepatocellular carcinoma in Egyptian patients. Arch Med Sci 2019; 15: 1454-61.

20. Kraus VB, Burnett B, Coindreau J, et al. Application of biomarkers in the development of drugs intended for the treatment of osteoarthritis. Osteoarthritis Cartilage 2011; 9: 515-42

21. Szemraj-Rogucka Z, Szemraj J, Masiarek K, Majos A Circulating microRNAs as biomarkers for myocardial fibrosis in patients with left ventricular non-compaction cardiomyopathy. Arch Med Sci 2019; 15: 376-84.

22. Volck B, Johansen JS, Stoltenberg $M$, et al. Studies on YKL-40 in knee joints of patients with rheumatoid arthritis and osteoarthritis. Involvement of YKL-40 in the joint pathology. Osteoarthritis Cartilage 2001; 9: 203-14.

23. Gracie JA, Forsey RJ, Chan WL, et al. A proinflammatory role for IL-18 in rheumatoid arthritis. J Clin Invest 1999; 104: 1393-401.

24. Dai S, Shan Z, Nishioka K, Yudoh K. Implication of interleukin 18 in production of matrix metalloproteinases in articular chondrocytes in arthritis: direct effect on chondrocytes may not be pivotal. Ann Rheum Dis 2005; 64: 735-42.

25. Rasmy H, Mikhael N, Ismail S. Interleukin-18 expression and the response to treatment in patients with psoriasis. Arch Med Sci 2011; 7: 713-9.

26. Fu ZZ, Liu P, Yang DH, et al. Interleukin-18-induced inflammatory responses in synoviocytes and chondrocytes from osteoarthritic patients. Int J Mol Med 2012; 30: 805-10.

27. Blumberg H, Conklin D, Xu WF, et al. Interleukin 20: discovery, receptor identification, and role in epidermal function. Cell 2001; 104: 9-19.

28. Hsu YH, Yang YY, Huwang MH, et al. Anti-IL-20 monoclonal antibody inhibited inflammation and protected against cartilage destruction in murine models of osteoarthritis. PLoS One 2017; 12: e0175802.

29. Hsu YH, Chang MS. IL-20 in rheumatoid arthritis. Drug Discov Today 2015; 22: 960-4.
30. Hsu YH, Li HH, Hsieh MY, et al. Function of interleukin-20 as a proinflammatory molecule in rheumatoid and experimental arthritis. Arthritis Rheum 2006; 54: 2722-33.

31. Li HH, Hsu YH, Wei CC, Lee PT, Chen WC, Chang MS. Interleukin-20 induced cell death in renal epithelial cells and was associated with acute renal failure. Genes Immun 2008; 9: 395-404.

32. Hsu YH, Chang MS. The therapeutic potential of anti-interleukin-20 monoclonal antibody. Cell Transplantation 2014; 23: 631-9.

33. Hsu YH, Chang MS. Interleukin-20 antibody is a potential therapeutic agent for experimental arthritis. Arthritis Rheum 2010; 62: 3311-21.

34. Wei CC, Li HH, Hsu YH, Hsing CH, Sung JM, Chang MS. Interleukin-20 targets renal cells and is associated with chronic kidney disease. Biochem Biophys Res Commun 2008; 374: 448-53.

35. Tritsaris K, Myren M, Ditlev SB, et al. IL-20 is an arteriogenic cytokine that remodels collateral networks and improves functions of ischemic hind limbs. Proc Natl Acad Sci USA 2007; 104: 15364-9.

36. Chen WY, Cheng BC, Jiang MJ, Hsieh MY, Chang MS. IL-20 is expressed in atherosclerosis plaques and promotes atherosclerosis in apolipoprotein E-deficient mice. Arterioscler Thromb Vasc Biol 2006; 26: 2090-5.

37. Wei CC, Chen WY, Wang YC, et al. Detection of IL-20 and its receptors on psoriatic skin. Clin Immunol 2005; 117: 65-72.

38. Altman R, Asch E, Bloch D, et al. Development of criteria for the classification and reporting of osteoarthritis. Classification of osteoarthritis of the knee. Diagnostic and Therapeutic Criteria Committee of the American Rheumatism Association. Arthritis Rheum 1986; 29: 1039-49.

39. Lequesne MG. The algofunctional indices for hip and knee osteoarthritis. J Rheumatol 1997; 24: 779-81.

40. Bellamy N, Buchanan WW, Goldsmith CH, Campbell J, Stitt LW. Validation study of WOMAC: a health status instrument for measuring clinically important patient relevant outcomes to antirheumatic drug therapy in patients with osteoarthritis of the hip or knee. J Rheumatol 1988; 15: 1833-40.

41. Barr S, Bellamy N, Buchanan WW, et al. A comparative study of signal versus aggregate methods of outcome measurement based on the WOMAC osteoarthritis index. J Rheumatol 1994; 21: 2106-12.

42. Wolfe F, Kong SX. Rasch analysis of the Western Ontario MacMaster questionnaire (WOMAC) in 2205 patients with osteoarthritis, rheumatoid arthritis, and fibromyalgia. Ann Rheum Dis 1999; 58: 563-8.

43. Kellgren JH, Lawrence JS. Radiological assessment of osteoarthrosis. Ann Rheum Dis 1957; 16: 494-502.

44. Smeets RL, van de Loo FA, Arntz OJ, Bennink MB, Joosten $L A$, van den Berg WB. Adenoviral delivery of IL-18 binding protein $\mathrm{C}$ ameliorates collagen-induced arthritis in mice. Gene Ther 2003; 10: 1004-11.

45. Inoue H, Hiraoka K, Hoshino T, et al. High levels of serum IL-18 promote cartilage loss through suppression of aggrecan synthesis. Bone 2008; 42: 1102-10.

46. Scrivo R, Conigliaro P, Riccieri V, et al. Distribution of interleukin-10 family cytokines in serum and synovial fluid of patients with inflammatory arthritis reveals different contribution to systemic and joint inflammation. Clin Exp Immunol 2015; 179: 300-8.

47. Monibi F, Roller BL, Stoker A, Garner B, Bal S, Cook JL. identification of synovial fluid biomarkers for knee os- 
teoarthritis and correlation with radiographic assessment. J Knee Surg 2016; 29: 242-7.

48. Livshits G, Zhai G, Hart DJ, et al. Interleukin-6 is a significant predictor of radiographic knee osteoarthritis: the Chingford Study. Arthritis Rheum 2009; 60: 2037-45.

49. Doss F, Menard J, Hauschild M, et al. Elevated IL-6 levels in the synovial fluid of osteoarthritis patients stem from plasma cells. Scand I Rheumatol 2007; 36: 136-9.

50. Bellucci F, Meini S, Cucchi P, et al. Synovial fluid levels of bradykinin correlate with biochemical markers for cartilage degradation and inflammation in knee osteoarthritis. Osteoarthritis Cartilage 2013; 21: 1774-80.

51. El-Arman MM, El-Fayoumi G, El-Shal E, El-Boghdady I, El-Ghaweet A. Aggrecan and cartilage oligomeric matrix protein in serum and synovial fluid of patients with knee osteoarthritis. HSS J 2010; 6: 171-6.

52. Larsson S, Lohmander LS, Struglics A. Synovial fluid level of aggrecan ARGS fragments is a more sensitive marker of joint disease than glycosaminoglycan or aggrecan levels: a crosssectional study. Arthritis Res Ther 2009; 11: R92.

53. Bleasel J, Poole R, Heinegard D. Changes in serum cartilage marker levels indicate altered cartilage metabolism in families with osteoarthritis-related type II collagen gene mutation. Arthritis Rheum 1999; 42: 39-45.

54. Senolt L, Leszczynski P, Dokoupilova E, et al. Efficacy and safety of anti-interleukin-20 monoclonal antibody in patients with rheumatoid arthritis: a randomized phase Ila trial. Arthritis Rheumatol 2015; 67: 1438-48.

55. Zivanović S, Rackov LP, Vojvodić D, Vucetić D. Human cartilage glycoprotein 39- biomarker of joint damage in knee osteoarthritis. Int Orthop 2009; 33: 1165-70. 